\title{
Semantic Web Framework for Knowledge-Centric Clinical Decision Support Systems
}

\author{
Sajjad Hussain, Samina Raza Abidi, and Syed Sibte Raza Abidi \\ NICHE Research Group, Faculty of Computer Science, Dalhousie University, \\ Halifax, CANADA B3H 1 W5 \\ \{hussain, abidi, sraza\}@cs.dal.ca
}

\begin{abstract}
Lately, there have been considerable efforts to computerize Clinical Practice Guidelines (CPG) so that they can be executed via Clinical Decision Support Systems (CDSS) at the point of care. We present a Semantic Web framework to both model and execute the knowledge within a CPG to develop knowledge-centric CDSS. Our approach entails knowledge modeling through a synergy between multiple ontologies-i.e. a domain ontology, CPG ontology and patient ontology. We develop decision-rules based on the ontologies, and execute them with a proof engine to derive CPG-based patient specific recommendations. We present a prototype of our CPG-based CDSS to execute the CPG for Follow-up after Treatment for Breast Cancer.
\end{abstract}

Keywords: Clinical Decision Support Systems, Semantic Web, Ontologies, Clinical Practice Guidelines, Breast Cancer.

\section{Introduction}

Clinical Practice Guidelines (CPG) are evidence-based recommendations to assist clinical decision-making [1]. Clinical Decision Support Systems (CDSS), developed using CPG-mediated knowledge, can offer the functionality to (a) Execute the CPG at the point of care; (b) Guide healthcare practitioners to make evidence based decisions, actions and recommendations; (c) standardize the delivery of care at a particular healthcare setting; and (d) Collect all necessary and relevant patient data.

From a technical perspective, the key challenges in developing CPG-guided CDSS are: (i) The capture of the disease-specific knowledge inherent within a CPG, whilst maintaining the underlying semantics, avoiding ambiguities and identifying the key decisional elements; (ii) The specification of the interactions between the key CPG elements to realize an executable CPG plan; (iii) The transformation of the CPG decision logic into medically salient and executable logic-based decision rules; (iv) The execution of a computerized CPG based on both acquired and inferred patient information; and (v) The explanation of the CDSS recommendations in order to establish 'trust' with the user.

The emerging Semantic Web [2] approach offers interesting and pragmatic solutions to model CPG for developing CPG-guided CDSS. The Semantic Web purports the semantic modeling and markup of knowledge, its properties and its relations using well-defined semantics such as formal definitions of terms, ontological 
definitions of domain concepts and resources. For developing CDSS, the Semantic Web offers a logic-based framework to (a) semantically model the structure of a paper-based CPG to a semantically enriched formalism, such as a CPG ontology; (b) annotate the CPG, based on the CPG ontology elements, in terms of the Resource Description Framework (RDF); (c) represent the underlying clinical concepts and relationships inherent within a CPG in terms of a domain ontology, using the Web Ontology Language (OWL); (d) represent the different patient data sources in a semantically enriched formalism; (e) specify the CPG based decision-making logic in terms of symbolic rules, represented as N3 triples, that can be executed using proof engines; (f) ensure interoperability between multiple ontologically defined knowledge resources; and $(\mathrm{g})$ provide a justification trace of the inferred recommendations.

In this paper, we present a Semantic Web based framework for computerizing CPG to develop a CDSS. We demonstrate the modeling of a Breast Cancer Follow-up (BCF) CPG, leading to the development of a BCF-CDSS that is deployed at the point of care in primary care settings. Figure 1 shows the architecture of our Semantic Web based CDSS. Our CDSS development framework constitutes three key elements:

a) Modelling the overall declarative and procedural knowledge required for decision support. We developed three independent, yet interacting ontologies as follows: (i) A CPG Ontology that models the computerized structure of the CPG in terms of the Guideline Element Model (GEM) [3]; (ii) A Domain Ontology that models the medical knowledge pertaining to the domain of the CPG. The Domain Ontology represents both the CPG's concepts and relationships between these concepts as OWL classes and properties, respectively; and (iii) A Patient Ontology that models the patient in terms of the longitudinal medical record of patient. We used Protégé [4] to develop all the ontologies.

b) Authoring of CPG-medicated decision rules using the CPG Rule Authoring Module. We developed a simple rule syntax to author rules.

c) Execution of the CPG to provide case-specific decision support. The CPG Execution Module, built using the JENA-a logic-based proof engine [5], allows the execution of decision logic rules based on patient data. A novel feature of our CDSS is that it provides a justification trace of all inferred recommendations.

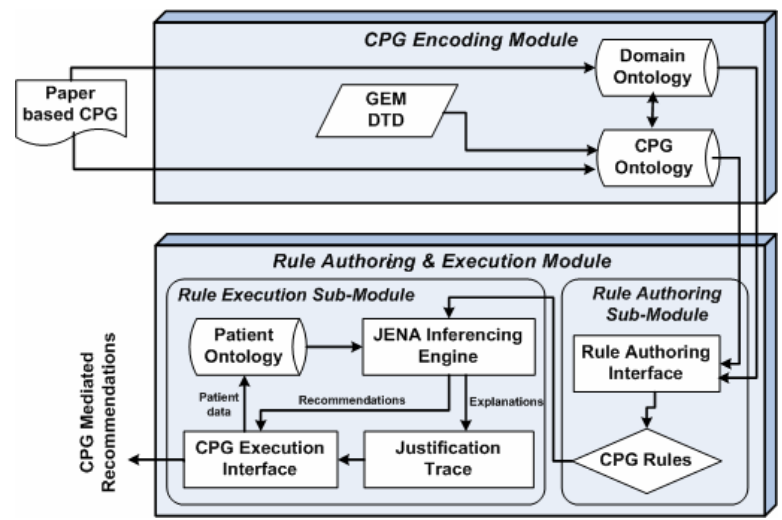

Fig. 1. Functional architecture of our CPG-Based CDSS 


\section{Clinical Practice Guideline Modeling Via Ontologies}

The Canadian Steering Committee on CPG for the Care and Treatment of Breast Cancer (BC) has developed and updated the guideline on follow-up care after treatment for $\mathrm{BC}$ [6], with a special emphasis on the needs of primary care physicians. The challenge was to operationalize the BCF-CPG within the family physician's clinical workflow so that $\mathrm{BC}$ follow-up can be offered in a primary care setting.

Computerization of the BCF-CPG was achieved using GEM, whereby the main task was to determine the function of a specific CPG text and annotate it using the relevant GEM tag. Next, we developed a Domain Ontology that models the knowledge encapsulated within the BCF-CPG. We used Protégé ontology editing environment to build our BC ontology using Protégé OWL. We defined twelve main classes, namely; Patient_Type, Physician_Type, Illness, Menstrual_Status, Recommendation, Symptom, Diagnostic_Tests, Treatment, Age, Risk_Factor, Weight_Status and Patient_Wish. Full details of the BC ontology are reported in [7].

\section{CPG Modeling Module}

We developed a CPG encoding tool that computerizes a paper-based CPG so that it can be executed by our CPG execution engine. The CPG encoding module comprises two components: (a) A GEM-based CPG representation formalism to convert the paper-based CPG into an electronic format (described earlier); and (b) A CPG ontology to model the structure of the CPG.

Our CPG Ontology is based on the GEM DTD [3]. The main CPG knowledge is represented in the Knowledge Component (KC) class in the CPG Ontology as they describe the procedural, conditional or imperative knowledge (as shown in Figure 2). We defined a Recommendation class to describe the recommended actions that are classified as being either imperative or conditional. Imperative recommendations are applicable to the entire eligible population, whereas conditional recommendations describe the clinical conditions/scenarios that demand specific actions. We represent these clinical conditions as decision.variable class in the CPG Ontology. We also made use of the property logic to define the decision logic for all recommendations based on the conditions for the various actions. Each decision.variable instance with a property variable.name is annotated with a property from the Domain Ontology.

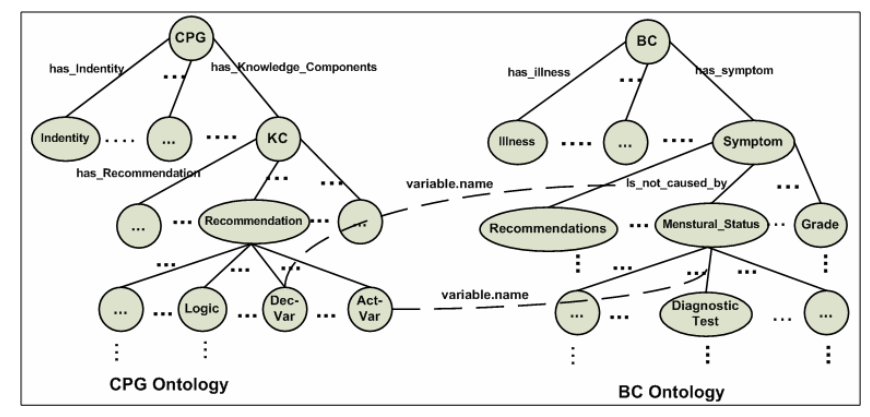

Fig. 2. Relation between CPG and Domain Ontology 


\section{CPG Authoring and Execution Module}

The CPG Rule Authoring and Execution module is designed to encapsulate the clinical decision logic inherent within a CPG in terms of logical rules-such logical rules are executed by a reasoning engine to derive $\mathrm{CPG}$-based recommendations. To achieve the above functionality, we built three sub-modules as follows:

\subsection{Rule Authoring Sub-module}

Rule Authoring is performed by defining decision rules in the logic tag of CPG ontology as follows: Step 1: Select decision variables, which represents the body (premises) of the rule; Step 2: Select the action variable, which represents the head (conclusion) of the rule; Step 3: For each decision variable and action variable in the rule, an equality/inequality relation can be defined with either a variable, a value, a binary algebraic formula, another decision variable or list of decision variables. An example conditional recommendation from the BCF-CPG "When such bleeding (Vaginal Bleeding) is present in the absence of obvious cause, endometrial biopsy should be carried out", can be defined in terms of a CPG decision rule via following rule authoring steps:

Step \# 1: First, we identified conditions in the above recommendation (marked as underline), and defined them as decision variables dvl=has_symptom, $d v 8=i s \_n o t \_c a u s e \_b y, d v 9=m s \_a p p l y \_t o \_d i a g n o s t i c \_t e s t$ and $a l=i s \_R e c o m m e n d e d$

Step \# 2: Then, we defined a rule in the logic tag of CPG Ontology by selecting decision and action variables that serve as conditions/premises and conclusion, respectively.

$$
\text { IF dv1, dv8, dv9 THEN a1 }
$$

Step \# 3: Finally, we defined this rule for a general case by quantifying decision variables for all such scenarios (represented as ?), as follows:

$$
\text { IF dv1=?,dv8=?,dv9=? THEN a1=[dv9] }
$$

Upon completion of the rule authoring process, we apply a rule transformation algorithm to transform the CPG rules into the JENA syntax so that they can be executed in the Execution Sub-Module that uses the JENA inference engine [5]. The above CPG rule $\mathrm{R}$ is transformed into JENA syntax via our rule transformation algorithm as follows:

Transform $(\mathrm{R})=$ [conditional1: (?X2 bc:has_symptom ?X5), (?X5 bc:is_not_cause_by ?X1), (?X1 bc:ms_apply_to_diagnostic_test ?X6) -> (?X2 bc:is_Recommended List(?X6 ))]

\subsection{Execution Sub-module}

The Execution Sub-Module invokes the JENA inference engine to execute a CPGi.e. infer recommendations based on patient data. We model instances from the Domain Ontology, CPG Ontology and Patient Ontology as RDF graphs, which serve as the knowledge base for JENA. The JENA inference engine employs backward reasoning to infer CPG-mediated recommendations based on the given patient scenario, encoded clinical knowledge in the Domain Ontology and CPG Ontology. 


\subsection{Justification Trace Sub-module}

Justification Trace Sub-Module generates a justification trace of the rule execution to assist medical practitioners in understanding the rationale behind the inferred recommendations. The justification derivation includes the linear representation of CPG rules that were satisfied to derive the stated recommendation. The justification trace initiates with an inferred patient recommendation (derived facts) and generates facts which served as premises for deriving the patient recommendation, recursively. The process terminates, if all the premises are ground instances (known facts). Below we show the justification trace for recommending Endometrial Biopsy for a BC patient Jane, who has symptom of Vaginal Bleeding.

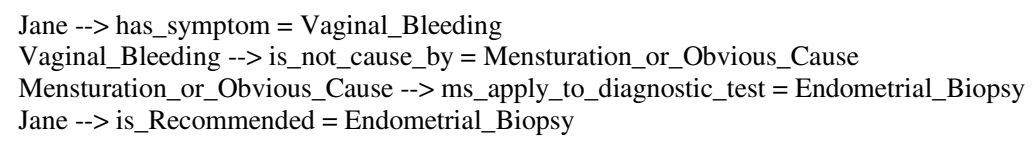

\section{Concluding Remarks}

In the realm of healthcare knowledge management the modeling of CPG provides interesting opportunities to develop CDSS that support evidence-guided recommendations. In this paper, we demonstrated the application of the Semantic Web to integrate multiple ontologies to develop a CDSS. Our CDSS approach is quite generic and can be extended to other medical problems. We tested our CDSS with a number of real-life clinical cases and both the recommendations and their justifications were validated by medical practitioners.

Acknowledgement. This project is supported by a research grant from Nova Scotia Health Research Foundation. Thanks to Dr. Grunfeld for the Breast Cancer CPG [6].

\section{References}

1. Field, M.J., Lohr, K.N. (eds.): Clinical Practice Guidelines: Directions for a New Program, Institute of Medicine, National Academy Press, Washington, DC (1990)

2. Berners-Lee, T.: The semantic web. Scientific American 284(5), 34-43 (2001)

3. Shiffman, R.N., Karras, B.T., Agrawal, A., Chen, R., Marenco, L., Nath, S.: GEM: A proposal for a more comprehensive guideline document model using XML. J Am Med Informatics Assoc 7, 488-498 (2000)

4. Knublauch, H., Fergerson, R., Noy, N.F., Musen, M.: The Protégé OWL Plugin: An open development environment for semantic web applications. In: McIlraith et al., pp. 229-243

5. JENA: Semantic Web Framework http://jena.sourceforge.net/documentation.html

6. Grunfeld, E., Dhesy-Thind, S., Levine, M.: Clinical practice Guidelines for the Care and Treatment of Breast Cancer: 9. Follow-up After Treatment for Breast Cancer (2005 update) Can. Med. Assoc. J 172, 1319-1320 (2005)

7. Abidi, S.: Ontology-based Modeling of Breast Cancer Follow-up Clinical Practice Guideline for Providing Clinical Decision Support. In: 20th IEEE Symposium on Computer-Based Medical Systems, Maribor, Slovenia, June 20-22, 2007, IEEE Press, Los Alamitos (2007) 